\title{
ElNoury - Webster bundle: a pre-emptive surgical approach for the management of morbidly low or adherent placenta
}

\author{
Amr Elnouri ${ }^{1}$, Dr Sophia Webster ${ }^{2}$, and Diaa Abdelhalim ${ }^{3}$ \\ ${ }^{1}$ Cairo University \\ ${ }^{2}$ Newcastle Upon Tyne Hospitals NHS Foundation Trust \\ ${ }^{3}$ El-Galaa Teaching Hospital
}

November 23, 2020

\begin{abstract}
Placenta accreta spectrum and placenta praevia are a significant cause of obstetric haemorrhage, maternal morbidity and mortality worldwide. We report a novel surgical technique, which pre-emptively and prophylactically reduces intrapartum bleeding during caesarean sections for these conditions and hence reducing the risk for a caesarean hysterectomy. This technique is particularly useful in low resource healthcare settings where interventional radiology is not readily available and where the woman is keen on uterine preservation. In this report we present the surgical and clinical outcomes of a case series of 16 patients on whom this technique was piloted demonstrating its feasibility and safety.
\end{abstract}

\section{Hosted file}

ELNOURY et al Manuscript Final.pdf available at https://authorea.com/users/378096/articles/ 494666-elnoury-webster-bundle-a-pre-emptive-surgical-approach-for-the-management-ofmorbidly-low-or-adherent-placenta 
Table 1. Demographic and operative details of the case series participants.

\begin{tabular}{|c|c|c|c|c|c|c|c|c|c|c|c|c|}
\hline $\begin{array}{l}\mathscr{\nu} \\
\tilde{d}\end{array}$ & 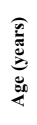 & 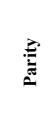 & 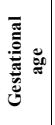 & 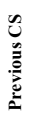 & 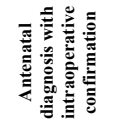 & 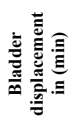 & 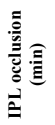 & 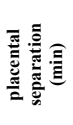 & 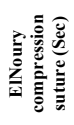 & 氞 & 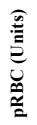 & 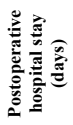 \\
\hline 1 & 36 & $\mathrm{G} 2 \mathrm{P} 1$ & 37 & 1 & Accreta + PP & 20 & 10 & 5 & 60 & 800 & 0 & 2 \\
\hline 2 & 35 & $\mathrm{G} 2 \mathrm{P} 1$ & 39 & 1 & Accreta + PP & 10 & 18 & 8 & 60 & 1000 & 0 & 2 \\
\hline 3 & 34 & G6P5 & 36 & 5 & Accreta + PP & 12 & 12 & 6 & 60 & 1400 & 2 & 2 \\
\hline 4 & 35 & G3P2 & 36 & 2 & Accreta + PP & 9 & 10 & 5 & 60 & 1300 & 2 & 2 \\
\hline 5 & 34 & G3P2 & 36 & 2 & Accreta + PP & 20 & 8 & 3 & 60 & 1400 & 3 & 1 \\
\hline 6 & 30 & G5P3 & 37 & 1 & Accreta + PP & 6 & 12 & 2 & 120 & 900 & 0 & 1 \\
\hline 7 & 24 & $\mathrm{G} 2 \mathrm{P} 1$ & 38 & 1 & PP & 7 & 6 & 3 & 60 & 700 & 0 & 1 \\
\hline 8 & 30 & G6P4 & 37 & 4 & Percreta + PP & 8 & 10 & 6 & 120 & 1400 & 5 & 3 \\
\hline 9 & 36 & G3P2 & 36 & 2 & Accreta + PP & 6 & 6 & 4 & 60 & 700 & 0 & 2 \\
\hline 10 & 38 & G4P3 & 36 & 3 & Increta + PP & 3 & 18 & 5 & 60 & 750 & 0 & 3 \\
\hline 11 & 38 & G5P4 & 36 & 4 & Accreta + PP & 15 & 15 & 13 & 80 & 750 & 0 & 1 \\
\hline 12 & 34 & G4P1 & 36 & 1 & Increta + PP & 8 & 14 & 6 & 80 & 2000 & 3 & 2 \\
\hline 13 & 31 & G3P2 & 36 & 2 & Percreta + PP & 20 & 12 & 3 & 80 & 800 & 0 & 2 \\
\hline 14 & 36 & G5P4 & 36 & 4 & Accreta + PP & 6 & 18 & 5 & 60 & 2500 & 4 & 3 \\
\hline 15 & 30 & G3P2 & 37 & 2 & Accreta + PP & 5 & 9 & 2 & 50 & 2000 & 4 & 2 \\
\hline 16 & 31 & $\mathrm{G} 2 \mathrm{P} 1$ & 37 & 1 & Accreta + PP & 5 & 15 & 4 & 70 & 800 & 0 & 2 \\
\hline
\end{tabular}

${ }^{*}$ No: Case number; ${ }^{*} \mathrm{GA}$ : gestational age (week); ${ }^{*} \mathrm{G} / \mathrm{P}$ : Gravidity/Parity; ${ }^{*} \mathrm{CS}$ : number of previous cesarean section; ${ }^{*}$ PP: placenta praevia; ${ }^{*} \mathrm{PL}$ : infundibulopelvic ligament; ${ }^{*} \mathrm{EBL}$ : estimated blood loss; *pRBC: transfused units of packed red blood cells intraoperative and postoperative 
Figure 1: Urinary bladder dissection
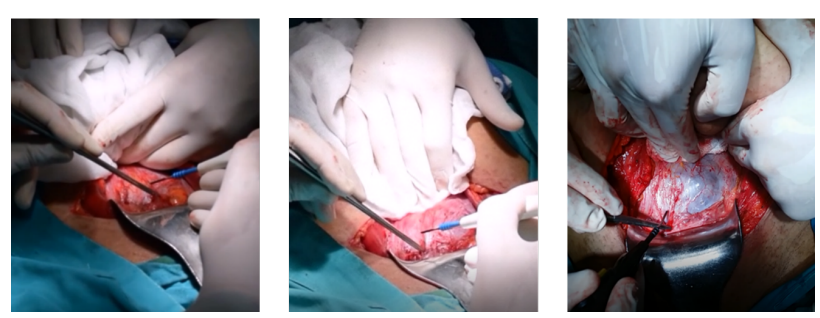
Figure 2: Temporary occlusion of the blood supply to the uterus
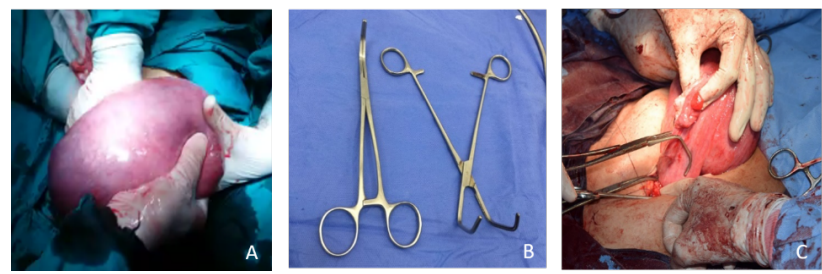

(A) Exteriorization of the uterus and Uterine artery compression by an assistant from behind uterus. (B) Satensky Vascular occluding clamp. (C) Application of Satensky vascular occluding clamp to infundibulo-pelvic ligaments. 


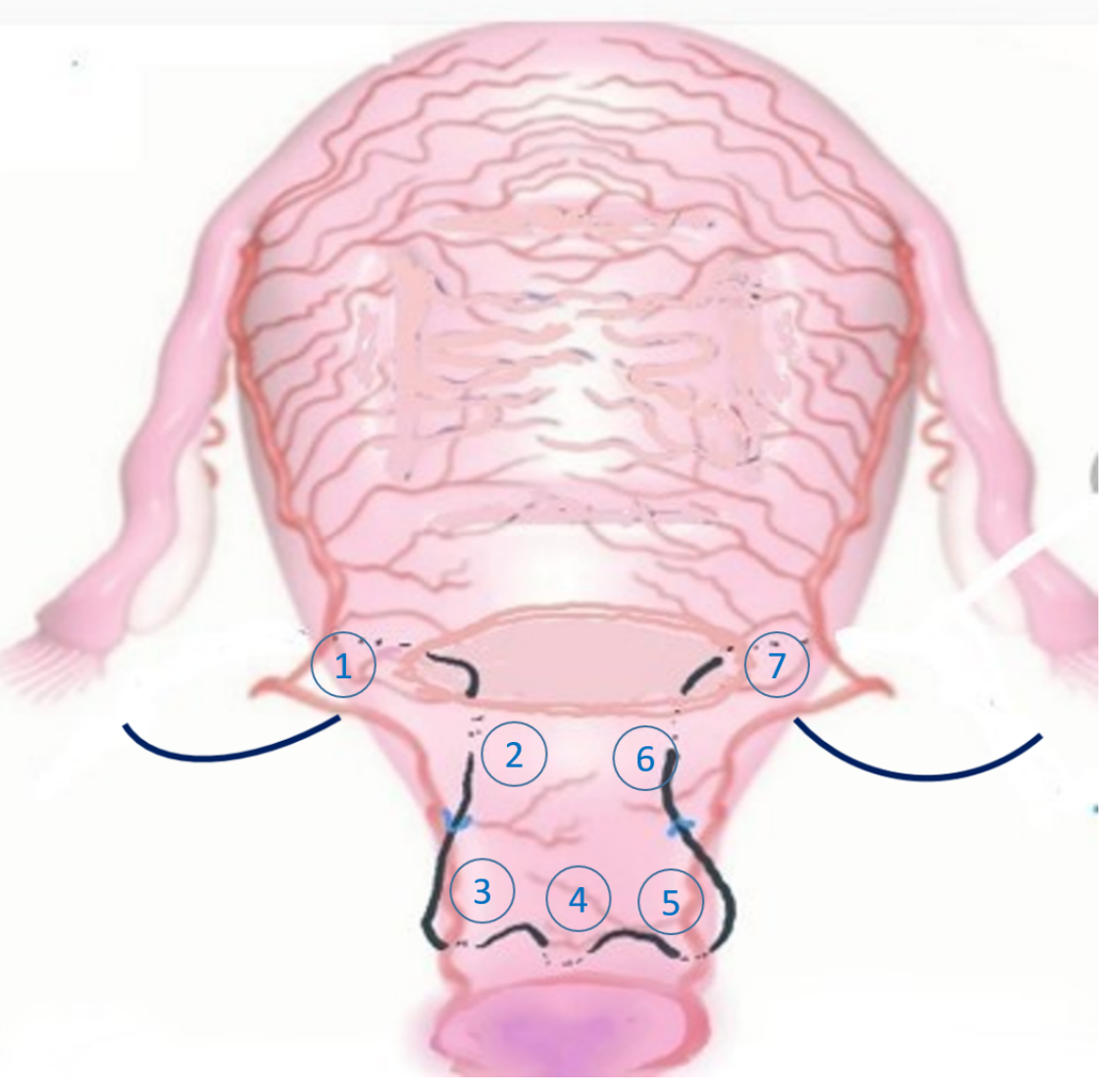

